\title{
The Bi-Integrable Couplings of Two-Component Casimir-Qiao-Liu Type Hierarchy and Their Hamiltonian Structures
}

\author{
Juhui Zhang and Yuqin Yao \\ Department of Applied Mathematics, China Agricultural University, Beijing 100083, China \\ Correspondence should be addressed to Yuqin Yao; yyqinw@126.com
}

Received 13 March 2016; Accepted 29 June 2016

Academic Editor: Boris G. Konopelchenko

Copyright (C) 2016 J. Zhang and Y. Yao. This is an open access article distributed under the Creative Commons Attribution License, which permits unrestricted use, distribution, and reproduction in any medium, provided the original work is properly cited.

\begin{abstract}
A new type of two-component Casimir-Qiao-Liu type hierarchy (2-CQLTH) is produced from a new spectral problem and their biHamiltonian structures are constructed. Particularly, a new completely integrable two-component Casimir-Qiao-Liu type equation (2-CQLTE) is presented. Furthermore, based on the semidirect sums of matrix Lie algebras consisting of $3 \times 3$ block matrix Lie algebra, the bi-integrable couplings of the 2-CQLTH are constructed and their bi-Hamiltonian structures are furnished.
\end{abstract}

\section{Introduction}

Solitons and integrable systems play an important role in nonlinear wave and dynamics systems. It has been significant in soliton theory to find more new integrable systems. In 1996, Olver and Rosenau obtained a Casimir equation [1]:

$$
\rho_{t}=\left(D \pm D^{3}\right) \rho^{-2}
$$

which is an integrable case of the general class of equations $\rho_{t}=D\left(1 \pm D^{2}\right) \rho^{k}$. In 2009, Qiao and Liu proposed a thirdorder integrable peakon equation $[2,3]$ :

$$
q_{t}=\left(\frac{1}{2 q^{2}}\right)_{x x x}-\left(\frac{1}{2 q^{2}}\right)_{x}
$$

which possesses Lax representation and bi-Hamiltonian structures. Here we can find that (2) can be obtained from the Casimir equation (1) by setting $\rho=\sqrt[3]{2} q$. We will use Casimir-Qiao-Liu equation (CQLE) to denote (2) in this paper. The CQLE (2) represents a third-order approximation of long wavelength, small amplitude waves of inviscid and incompressible fluids. Moreover, it may be reduced from the two-dimensional Euler equation by an approximation procedure, and its solutions may be useful to construct new solvable potentials in Newtonian dynamics and to model electrophysiological phenomena in neuroscience. It has attracted many scholars' attention in recent years [4-6].

In recent years, the construction of soliton hierarchies and integrable couplings have become important research fields in soliton theory [7-10]. The soliton hierarchies are generated from the zero curvature equations [11, 12] which are based on semisimple Lie algebras, while the integrable couplings are generated from the zero curvature equations based on semidirect sums of Lie algebras [13-15].

The trace identity proposed by $\mathrm{Tu}$ is a useful tool for constructing the Hamiltonian structures for both continuous and discrete integrable systems [11, 12]. Many integrable Hamiltonian systems of infinite dimensions with various physics and mathematical backgrounds have been obtained [16-18]. But when it comes to the integrable couplings, since they are based on semidirect sums of Lie algebras, the trace identity cannot be used properly. In order to solve this problem, Ma proposes the variational identity [19] while Guo and Zhang propose the quadratic-form identity [20]. By using them, the Hamiltonian structures of many integrable couplings systems have been furnished [21-23].

Integrable couplings correspond to nonsemisimple Lie algebras $\bar{g}$, and such Lie algebras can be written as semidirect sums [24]:

$$
\bar{g}=g \oplus g_{c}, \quad g \text {-semisimple, } g_{c} \text {-solvable. }
$$


The notion of semidirect sums $\bar{g}=g \oplus g_{c}$ means that the two Lie subalgebras $g$ and $g_{c}$ satisfy

$$
\left[g, g_{c}\right] \subseteq g_{c},
$$

where $\left[g, g_{c}\right]=\left\{[A, B] \mid A \in g, B \in g_{c}\right\}$, with $[\cdot, \cdot]$ denoting the Lie bracket of $\bar{g}$. We also require the closure property between $g$ and $g_{c}$ under the matrix multiplication:

$$
g g_{c}, g_{c} g \subseteq g_{c},
$$

where $g_{1} g_{2}=\left\{[A, B] \mid A \in g_{1}, B \in g_{2}\right\}$.

Now we make the following assumptions:

$$
\begin{aligned}
& \bar{U}=U+U_{c}, \\
& \bar{V}=V+V_{c},
\end{aligned}
$$

where $U, V \in g, U_{c}, V_{c} \in g_{c}$ and $\bar{U}, \bar{V}$ satisfying (3), (4), and (5). In this condition, we can construct the enlarged spectral problem as

$$
\begin{aligned}
& \bar{\phi}_{x}=\overline{U \phi}=\bar{U}(\bar{u}, \lambda) \bar{\phi}, \\
& \bar{\phi}_{t}=\overline{V \phi}=\bar{V}\left(\bar{u}, \bar{u}_{x}, \ldots, \frac{\partial^{m_{0}} \bar{u}}{\partial x^{m_{0}}} ; \lambda\right) \bar{\phi} .
\end{aligned}
$$

From the enlarged zero curvature equations

$$
\bar{U}-\bar{V}_{x}+[\bar{U}, \bar{V}]=0,
$$

we have

$$
\begin{aligned}
U-V_{x}+[U, V] & =0, \\
U_{c, t}-V_{c, x}+\left[U, V_{c}\right]+\left[U_{c}, V\right]+\left[U_{c}, V_{c}\right] & =0 .
\end{aligned}
$$

The first equation of (9) is the original soliton hierarchy while the second equation is the integrable couplings.

The bi-Hamiltonian structures of (9)

$$
\bar{u}_{t_{m}}=\bar{J} \frac{\delta \mathscr{H}_{m+1}}{\delta \bar{u}}, \quad m \geq 0,
$$

can be obtained by using the following variational identity:

$$
\begin{aligned}
\frac{\delta}{\delta u} \int\left\langle V, \frac{\partial U}{\partial \lambda}\right\rangle d x=\lambda^{-\gamma} \frac{\partial}{\partial \lambda} \lambda^{\gamma}\left\langle V, \frac{\partial U}{\partial u}\right\rangle, & \\
\gamma & =-\frac{\lambda}{2} \frac{d}{d \lambda} \ln |\langle V, V\rangle|,
\end{aligned}
$$

where $\langle\cdot, \cdot\rangle$ is a bilinear form $[19,20,25]$.

In this paper, starting from a new eigenvalue problem, the two-component Casimir-Qiao-Liu type hierarchy (2CQLTH) is worked out. It is proved that the 2-CQLTH has Lax pairs and bi-Hamiltonian structures, so it is completely integrable. Particularly, the two-component Casimir-QiaoLiu type equation (2-CQLTE) is given. Then, by constructing the enlarged spectral problem, we obtain the the bi-integrable couplings of the 2-CQLTH. Similarly, we have proved the integrability of the bi-integrable couplings by constructing their Lax pairs and bi-Hamiltonian structures.
This paper is organized as follows. In Section 2, a new 2CQLTH is given. In Section 3, the bi-Hamiltonian structures of the 2-CQLTH are constructed. In Section 4, we obtain the bi-integrable couplings of the 2-CQLTH. In Section 5, we generate the bi-Hamiltonian structures of the integrable couplings. The conclusion is given in Section 6 .

\section{The Two-Component Casimir-Qiao-Liu Type Hierarchy}

Consider a spatial matrix isospectral problem:

$$
\begin{aligned}
& \phi_{x}=U \phi=U(u, \lambda) \phi, \\
& \quad U(u, \lambda)=\left[\begin{array}{cc}
-p & \frac{1}{2} \lambda q \\
-\frac{1}{2} \lambda q & p
\end{array}\right], u=\left[\begin{array}{l}
p \\
q
\end{array}\right], \phi=\left[\begin{array}{l}
\phi_{1} \\
\phi_{2}
\end{array}\right],
\end{aligned}
$$

where $p, q$ are potential functions and $\lambda$ is the spectral parameter.

Assuming that $W$ has the form

$$
W=\left[\begin{array}{cc}
a & b \\
c & -a
\end{array}\right],
$$

then the stationary zero curvature equation

$$
W_{x}=[U, W]
$$

gives rise to

$$
\begin{aligned}
& a_{x}=\frac{1}{2} b q \lambda+\frac{1}{2} c q \lambda, \\
& b_{x}=-a q \lambda-2 b p, \\
& c_{x}=-a q \lambda+2 c p .
\end{aligned}
$$

Further, let $a, b, c$ possess the Laurent expansions:

$$
\begin{aligned}
& a=\sum_{i \geq 0} a_{i} \lambda^{-2 i}, \\
& b=\sum_{i \geq 0} b_{i} \lambda^{-2 i-1}, \\
& c=\sum_{i \geq 0} c_{i} \lambda^{-2 i-1},
\end{aligned}
$$

$$
i \geq 0,
$$

where the initial values are as follows:

$$
\begin{aligned}
& a_{0}=0, \\
& b_{0}=\frac{1}{4}, \\
& c_{0}=-\frac{1}{4} .
\end{aligned}
$$


In this case, system (15) becomes

$$
\begin{aligned}
a_{i, x} & =\frac{q}{2}\left(b_{i}+c_{i}\right), \\
b_{i, x} & =-2 p b_{i}-q a_{i+1}, \\
c_{i, x} & =2 p c_{i}-q a_{i+1}, \\
& \quad i \geq 0 .
\end{aligned}
$$

Imposing the following conditions on constants of integration:

$$
\left.a_{i}\right|_{u=0}=\left.b_{i}\right|_{u=0}=\left.c_{i}\right|_{u=0}=0, \quad i \geq 1,
$$

the sequence of $\left\{a_{i}, b_{i}, c_{i} \mid i \geq 1\right\}$ can be uniquely determined and the first two sets are as follows:

$$
\begin{aligned}
& a_{1}=-\frac{p}{2 q} \\
& b_{1}=-\frac{1}{2 q}\left(\frac{p}{q}\right)_{x}+\frac{1}{2}\left(\frac{p}{q}\right)^{2}, \\
& c_{1}=-\frac{1}{2 q}\left(\frac{p}{q}\right)_{x}-\frac{1}{2}\left(\frac{p}{q}\right)^{2}, \\
& a_{2}=-\frac{p^{3}}{q^{3}}+\frac{p_{x x}}{2 q^{3}}-\frac{3 p_{x} q_{x}}{2 q^{4}}-\frac{p q_{x x}}{2 q^{4}}+\frac{3 p q_{x}^{2}}{2 q^{5}}, \\
& b_{2}=\frac{a_{2, x}}{q}-2 \int \frac{p}{q} a_{2, x} d x, \\
& c_{2}=\frac{a_{2, x}}{q}+2 \int \frac{p}{q} a_{2, x} d x .
\end{aligned}
$$

Now we introduce

$$
V^{[m]}=\lambda\left(\lambda^{2 m+1} W\right)_{+}+\Delta_{m}, \quad m \geq 0,
$$

where

$$
\Delta_{m}=\left[\begin{array}{cc}
-a_{m+1} & \lambda \frac{q}{p} a_{m+1} \\
-\lambda \frac{q}{p} a_{m+1} & a_{m+1}
\end{array}\right] .
$$

By considering

$$
\phi_{t_{m}}=V^{(m)} \phi
$$

the compatibility of (12) and (23) gives the zero curvature equation:

$$
U_{t_{m}}-V_{x}^{[m]}+\left[U, V^{[m]}\right]=0
$$

From the zero curvature equation (24), we can obtain the 2CQLTH:

$$
u_{t_{m}}=K_{m}=\left[\begin{array}{c}
a_{m+1, x} \\
2\left(\frac{q}{p} a_{m+1}\right)_{x}
\end{array}\right]=J\left[\begin{array}{c}
-4 a_{m+1} \\
c_{m+1}-b_{m+1}
\end{array}\right],
$$

where

$$
J=\left[\begin{array}{cc}
-\frac{1}{4} \partial & 0 \\
0 & \frac{1}{2} \partial \frac{q}{p} \partial^{-1} \frac{q}{p} \partial
\end{array}\right]
$$

Remark 1. The Lax pair for the 2-CQLTH (see (25)) is given by (12) and (23). This implies that the 2-CQLTH (see (25)) is integrable in Lax sense.

Particularly, when we take $m=1$, we obtain

$$
\begin{aligned}
p_{t} & =-\left(\frac{3 p^{3}}{q^{3}}+\frac{2 p q_{x x}}{q^{4}}-\frac{15 p q_{x}^{2}}{2 q^{5}}\right)\left(\frac{p_{x}}{p}-\frac{q_{x}}{q}\right) \\
& -\frac{3 p q_{x}}{q^{4}}\left(\frac{p_{x x}}{p}-\frac{q_{x x}}{q}\right)+\frac{p}{2 q^{3}}\left(\frac{p_{x x x}}{p}-\frac{q_{x x x}}{q}\right) \\
q_{t} & =-\left(\frac{4 p^{2}}{q^{2}}+\frac{p_{x x}}{p q^{2}}-\frac{3 p_{x} q_{x}}{p q^{3}}+\frac{3 q_{x x}}{q^{3}}-\frac{12 q_{x}^{2}}{q^{4}}\right) \\
& \cdot\left(\frac{p_{x}}{p}-\frac{q_{x}}{q}\right)-\frac{6 q_{x}}{q^{3}}\left(\frac{p_{x x}}{p}-\frac{q_{x x}}{q}\right) \\
& +\frac{1}{q^{2}}\left(\frac{p_{x x x}}{p}-\frac{q_{x x x}}{q}\right) .
\end{aligned}
$$

Remark 2. Taking $p=1 / 2$, the second equation of (27) can be reduced to the CQLE (2). Thus, (27) is called 2-CQLTE.

\section{Bi-Hamiltonian Structures and Liouville Integrability}

By direct computation, we can get

$$
\begin{aligned}
& \frac{\partial U}{\partial \lambda}=\left[\begin{array}{cc}
0 & \frac{1}{2} q \\
-\frac{1}{2} q & 0
\end{array}\right], \\
& \frac{\partial U}{\partial p}=\left[\begin{array}{cc}
-1 & 0 \\
0 & 1
\end{array}\right], \\
& \frac{\partial U}{\partial q}=\left[\begin{array}{cc}
0 & \frac{1}{2} \lambda \\
-\frac{1}{2} \lambda & 0
\end{array}\right],
\end{aligned}
$$

so we have

$$
\begin{aligned}
& \operatorname{tr}\left(W \frac{\partial U}{\partial \lambda}\right)=\frac{1}{2} q c-\frac{1}{2} q b, \\
& \operatorname{tr}\left(W \frac{\partial U}{\partial p}\right)=-2 a, \\
& \operatorname{tr}\left(W \frac{\partial U}{\partial q}\right)=\frac{1}{2} c \lambda-\frac{1}{2} b \lambda .
\end{aligned}
$$


Now, the trace identity [11]

$$
\begin{aligned}
\frac{\delta}{\delta u} \int \operatorname{tr}\left(W \frac{\partial U}{\partial \lambda}\right) d x=\lambda^{-\gamma} \frac{\partial}{\partial \lambda} \lambda^{\gamma} \operatorname{tr}\left(W \frac{\partial U}{\partial u}\right), \\
\gamma=\frac{\lambda}{2} \frac{d}{d \lambda} \ln \left|\operatorname{tr}\left(W^{2}\right)\right|
\end{aligned}
$$

gives

$$
\frac{\delta}{\delta u} \int\left(\frac{1}{2} q c-\frac{1}{2} q b\right) d x=\lambda^{-\gamma} \frac{\partial}{\partial \lambda} \lambda^{\gamma}\left[\begin{array}{c}
-2 a \\
\frac{1}{2} c \lambda-\frac{1}{2} b \lambda
\end{array}\right] .
$$

Comparing coefficients of all powers of $\lambda$ in the equality (31), we have

$$
\frac{\delta}{\delta u} \int \frac{q c_{m}-q b_{m}}{\gamma-2 m} d x=\left[\begin{array}{c}
-4 a_{m} \\
c_{m}-b_{m}
\end{array}\right], \quad m \geq 0 .
$$

Checking a particular case with $m=1$ in (32), we have $\gamma=1$.

Thus, we obtain

$$
\frac{\delta}{\delta u} \mathscr{H}_{m}=\left[\begin{array}{c}
-4 a_{m} \\
c_{m}-b_{m}
\end{array}\right], \quad m \geq 0,
$$

where the Hamiltonian functionals are defined by

$$
\mathscr{H}_{m}=\int \frac{q c_{m}-q b_{m}}{1-2 m} d x
$$

It now follows that the soliton hierarchy (25) has the following Hamiltonian structures:

$$
u_{t_{m}}=K_{m}=J \frac{\delta \mathscr{H}_{m}}{\delta u}, \quad m \geq 0 .
$$

From the recursion relations (18), we can get

$$
\left(\begin{array}{c}
-4 a_{m+1} \\
c_{m+1}-b_{m+1}
\end{array}\right)=L\left(\begin{array}{c}
-4 a_{m} \\
c_{m}-b_{m}
\end{array}\right), \quad m \geq 0,
$$

where

$$
=\left[\begin{array}{cc}
-\frac{1}{q} \partial \frac{1}{q} \partial+4 \frac{p}{q} \partial^{-1} \frac{p}{q} \partial & 0 \\
0 & -\partial^{-1} \frac{p}{q} \partial \frac{1}{q} \partial \frac{1}{p} \partial+4 \partial^{-1} \frac{p}{q} \partial \frac{p}{q}
\end{array}\right] .
$$

It is easy to verify that $J L=L^{*} J$. Actually, we know that the hierarchy (25) is bi-Hamiltonian:

$$
u_{t_{m}}=K_{m}=J \frac{\delta \mathscr{H}_{m}}{\delta u}=M \frac{\delta \mathscr{H}_{m-1}}{\delta u}, \quad m \geq 1,
$$

where the second Hamiltonian operator is

$$
\begin{aligned}
M & =J L \\
& =\left[\begin{array}{cc}
-\partial \frac{p}{q} \partial^{-1} \frac{p}{q} \partial+\frac{1}{4} \partial \frac{1}{q} \partial \frac{1}{q} \partial & 0 \\
0 & 2 \partial-\frac{1}{2} \partial \frac{1}{p} \partial \frac{1}{p} \partial
\end{array}\right]
\end{aligned}
$$

and $J$ and $M$ constitute the Hamiltonian pairs. Thus, the soliton hierarchy (25) is Liouville integrable.
Remark 3. Based on $\mathrm{SO}(3)[26,27]$, a $3 \times 3$ matrix isospectral problem is constructed:

$$
\phi_{x}=U \phi=U(u, \lambda) \phi, \quad u=\left[\begin{array}{l}
p \\
q
\end{array}\right], \phi=\left[\begin{array}{l}
\phi_{1} \\
\phi_{2}
\end{array}\right],
$$

with the spectral matrix as

$$
U=\left[\begin{array}{ccc}
0 & -\frac{q \lambda}{2} & -p \\
\frac{q \lambda}{2} & 0 & -\frac{q \lambda}{2} \\
p & \frac{q \lambda}{2} & 0
\end{array}\right]
$$

By a standard procedure similar to Sections 2 and 3, another 2-QLTH can be worked out:

$$
u_{t_{m}}=\left[\begin{array}{c}
-a_{m+1, x} \\
-2\left(\frac{q}{p} a_{m+1}\right)_{x}
\end{array}\right]=J \frac{\delta \mathscr{H}_{m}}{\delta u}=M \frac{\delta \mathscr{H}_{m-1}}{\delta u},
$$

$m \geq 1$,

with the Hamiltonian operators

$$
\begin{aligned}
J= & {\left[\begin{array}{cc}
-\partial \frac{1}{q} \partial \frac{1}{q} \partial-\partial \frac{p}{q} \partial^{-1} \frac{p}{q} \partial & 0 \\
0 & 2 \partial \frac{1}{p} \partial \frac{1}{p} \partial+2 \partial
\end{array}\right], } \\
M= & {\left[\begin{array}{cc}
M_{1} & 0 \\
0 & M_{2}
\end{array}\right], } \\
M_{1}= & 2 \partial \frac{1}{q} \partial \frac{1}{q} \partial \frac{1}{q} \partial \frac{1}{q} \partial+2 \partial \frac{1}{q} \partial \frac{1}{q} \partial \frac{p}{q} \partial^{-1} \frac{p}{q} \partial \\
& +2 \partial \frac{p}{q} \partial^{-1} \frac{p}{q} \partial \frac{1}{q} \partial \frac{1}{q} \partial+2 \partial \frac{p}{q} \partial^{-1} \frac{p}{q} \partial \frac{p}{q} \partial^{-1} \frac{p}{q} \partial, \\
M_{2}= & -4 \partial \frac{1}{p} \partial \frac{1}{q} \partial \frac{1}{q} \partial \frac{1}{p} \partial-4 \partial \frac{1}{p} \partial \frac{1}{q} \partial \frac{p}{q}-4 \frac{p}{q} \partial \frac{1}{q} \partial \frac{1}{p} \partial \\
& -4 \frac{p}{q} \partial \frac{p}{q},
\end{aligned}
$$

and the Hamiltonian functionals

$$
\mathscr{H}_{m}=\int \frac{-q b_{m}-q c_{m}}{1-2 m} d x, \quad m \geq 0 .
$$


Particularly, when we take $m=1$, a new 2-CQLTE is presented as

$$
\begin{aligned}
p_{t} & =\left(\frac{3 p^{3}}{4 q^{3}}-\frac{2 p q_{x x}}{q^{4}}+\frac{15 p q_{x}^{2}}{2 q^{5}}\right)\left(\frac{p_{x}}{p}-\frac{q_{x}}{q}\right)-3 \\
& \cdot \frac{p q_{x}}{q^{4}}\left(\frac{p_{x x}}{p}-\frac{q_{x x}}{q}\right)+\frac{p}{2 q^{3}}\left(\frac{p_{x x x}}{p}-\frac{q_{x x x}}{q}\right), \\
q_{t} & =\left(\frac{p^{2}}{q^{2}}-\frac{p_{x x}}{p q^{2}}-3 \frac{q_{x x}}{q^{3}}+3 \frac{p_{x} q_{x}}{p q^{3}}+12 \frac{q_{x}^{2}}{q^{4}}\right) \\
& \cdot\left(\frac{p_{x}}{p}-\frac{q_{x}}{q}\right)-6 \frac{q_{x}}{q^{3}}\left(\frac{p_{x x}}{p}-\frac{q_{x x}}{q}\right) \\
& +\frac{1}{q^{2}}\left(\frac{p_{x x x}}{p}-\frac{q_{x x x}}{q}\right) .
\end{aligned}
$$

Equation (45) can be transformed into (27) in complex field, but they are different equations in real field.

\section{Bi-Integrable Couplings of the 2-CQLTH}

Supposing that the triangular block matrices $M$ have the following form $[28,29]$ :

$$
M\left(A_{1}, A_{2}, A_{3}\right)=\left(\begin{array}{ccc}
A_{1} & A_{2} & A_{3} \\
0 & A_{1} & \delta A_{2} \\
0 & 0 & A_{1}
\end{array}\right),
$$

where $\delta$ is a constant, and defining $\bar{g}=\left\{M\left(A_{1}, A_{2}, A_{3}\right)\right\}, g=$ $\left\{M\left(A_{1}, 0,0\right)\right\}$, and $g_{c}=\left\{M\left(0, A_{2}, A_{3}\right)\right\}$, we know that $\bar{g}, g, g_{c}$ satisfy (3), (4), and (5).

Considering the enlarged spectral matrix

$$
\begin{aligned}
& \bar{U}=\left(\begin{array}{ccc}
U & U_{1} & U_{2} \\
0 & U & \delta U_{1} \\
0 & 0 & U
\end{array}\right), \\
& \bar{W}=\left(\begin{array}{ccc}
W & W_{1} & W_{2} \\
0 & W & \delta W_{1} \\
0 & 0 & W
\end{array}\right),
\end{aligned}
$$

where

$$
\begin{gathered}
U=\left(\begin{array}{cc}
-p & \frac{q \lambda}{2} \\
-\frac{q \lambda}{2} & p
\end{array}\right), \\
U_{1}=\left(\begin{array}{cc}
-r_{1} & \frac{r_{2} \lambda}{2} \\
-\frac{r_{2} \lambda}{2} & r_{1}
\end{array}\right),
\end{gathered}
$$

$$
\begin{aligned}
& U_{2}=\left(\begin{array}{cc}
-s_{1} & \frac{s_{2} \lambda}{2} \\
-\frac{s_{2} \lambda}{2} & s_{1}
\end{array}\right), \\
& W=\left(\begin{array}{cc}
a & b \\
c & -a
\end{array}\right), \\
& W_{1}=\left(\begin{array}{cc}
d & e \\
f & -d
\end{array}\right), \\
& W_{2}=\left(\begin{array}{cc}
g & h \\
k & -g
\end{array}\right),
\end{aligned}
$$

and $p, q, r_{1}, r_{2}, s_{1}, s_{2}$ are dependent variables, if we substitute $\bar{U}, \bar{W}$ into the corresponding enlarged stationary zero curvature equation, we can get

$$
\begin{aligned}
2 a_{x}-b q \lambda-c q \lambda & =0, \\
b_{x}+2 b p+a q \lambda & =0, \\
c_{x}-2 c p+a q \lambda & =0, \\
2 d_{x}-e q \lambda-f q \lambda-b \lambda r_{2}-c \lambda r_{2} & =0, \\
e_{x}+2 e p+d q \lambda+2 b r_{1}+a \lambda r_{2} & =0, \\
f_{x}-2 f p+d q \lambda-2 c r_{1}+a \lambda r_{2} & =0, \\
2 g_{x}-h q \lambda-k q \lambda-e \delta \lambda r_{2}-f \delta \lambda r_{2}-b \lambda s_{2}-c \lambda s_{2} & =0, \\
h_{x}+2 h p+g q \lambda+2 e \delta r_{1}+d \delta \lambda r_{2}+2 b s_{1}+a \lambda s_{2} & =0, \\
k_{x}-2 k p+g q \lambda-2 f \delta r_{1}+d \delta \lambda r_{2}-2 c s_{1}+a \lambda s_{2} & =0 .
\end{aligned}
$$

When $a, b, c, d, e, f, g, h, k$ are assumed to be

$$
\begin{aligned}
& a=\sum_{i \geq 0} a_{i} \lambda^{-2 i}, \\
& b=\sum_{i \geq 0} b_{i} \lambda^{-2 i-1}, \\
& c=\sum_{i \geq 0} c_{i} \lambda^{-2 i-1},
\end{aligned}
$$

$i \geq 0$,

$$
\begin{aligned}
& d=\sum_{i \geq 0} d_{i} \lambda^{-2 i}, \\
& e=\sum_{i \geq 0} e_{i} \lambda^{-2 i-1}, \\
& f=\sum_{i \geq 0} f_{i} \lambda^{-2 i-1},
\end{aligned}
$$

$i \geq 0$, 


$$
\begin{aligned}
& g=\sum_{i \geq 0} g_{i} \lambda^{-2 i}, \\
& h=\sum_{i \geq 0} h_{i} \lambda^{-2 i-1}, \\
& k=\sum_{i \geq 0} k_{i} \lambda^{-2 i-1},
\end{aligned}
$$

$i \geq 0$,

(50)

and the initial values are taken as

$$
\begin{aligned}
& a_{0}=0, \\
& b_{0}=\alpha, \\
& c_{0}=-\alpha, \\
& d_{0}=0, \\
& e_{0}=\beta, \\
& f_{0}=-\beta, \\
& g_{0}=0, \\
& h_{0}=\iota, \\
& k_{0}=-\iota,
\end{aligned}
$$

we can write (49) in the following forms:

$$
\begin{aligned}
& -\frac{1}{2} b_{i} q-c_{i} \frac{q}{2}+a_{i, x}=0 \\
& 2 b_{i} p+a_{i+1} q+b_{i, x}=0 \\
& -2 c_{i} p+a_{i+1} q \lambda+c_{i, x}=0 \\
& -\frac{1}{2} e_{i} q-f_{i} \frac{q}{2}+d_{i, x}-\frac{1}{2} b_{i} r_{2}-\frac{1}{2} c_{i} r_{2}=0, \\
& 2 e_{i} p+d_{i+1} q \lambda+e_{i, x}+2 b r_{1}+a_{i+1} r_{2}=0, \\
& -2 f_{i} p+d_{i+1} q+f_{i, x}-2 c_{i} r_{1}+a_{i+1} r_{2}=0, \\
& \quad-\frac{1}{2} h_{i} q-\frac{k_{i} q}{2}+g_{i, x}-\frac{1}{2} e_{i} \delta r_{2}-\frac{1}{2} f_{i} \delta r_{2}-\frac{1}{2} b_{i} s_{2} \\
& \quad-\frac{1}{2} c_{i} s_{2}=0, \\
& 2 h_{i} p+g_{i+1} q+h_{i, x}+2 e_{i} \delta r_{1}+d_{i+1} \delta r_{2}+2 b_{i} s_{1} \\
& \quad+a_{i+1} s_{2}=0, \\
& -2 k_{i} p+g_{i+1} q+k_{i, x}-2 f_{i} \delta r_{1}+d_{i+1} \delta \lambda r_{2}-2 c_{i} s_{1} \\
& \quad+a_{i+1} \lambda s_{2}=0 .
\end{aligned}
$$

Furthermore, by imposing

$$
\begin{aligned}
& \left.a_{i}\right|_{u=0}=\left.b_{i}\right|_{u=0}=\left.c_{i}\right|_{u=0}=0, \quad i \geq 1, \\
& \left.d_{i}\right|_{u=0}=\left.e_{i}\right|_{u=0}=\left.f_{i}\right|_{u=0}=0, \quad i \geq 1, \\
& \left.g_{i}\right|_{u=0}=\left.h_{i}\right|_{u=0}=\left.k_{i}\right|_{u=0}=0, \quad i \geq 1,
\end{aligned}
$$

the sequence of $\left\{a_{i}, b_{i}, c_{i}, d_{i}, e_{i}, f_{i}, g_{i}, h_{i}, k_{i} \mid i \geq 1\right\}$ has the unique expressions

$$
\begin{aligned}
& a_{0}=0, \\
& b_{0}=\alpha, \\
& c_{0}=-\alpha,
\end{aligned}
$$$$
a_{1}=-2 \alpha \frac{p}{q}
$$

$$
b_{1}=2 \alpha\left(\frac{p}{q}\right)^{2}-2 \alpha \frac{1}{q}\left(\frac{p}{q}\right)_{x}
$$$$
c_{1}=-2 \alpha\left(\frac{p}{q}\right)^{2}-2 \alpha \frac{1}{q}\left(\frac{p}{q}\right)_{x} \text {, }
$$

$d_{0}=0$,

$e_{0}=\beta$,

$f_{0}=-\beta$,

$d_{1}=-2 \alpha \frac{p}{q}-2 \beta \frac{r_{1}}{q}+2 \beta \frac{p r_{2}}{q^{2}}$,

$e_{1}=2 \alpha\left(\frac{p}{q}\right)^{2}-2 \alpha \frac{1}{q}\left(\frac{p}{q}\right)_{x}-2 \beta \frac{1}{q}\left(\frac{r_{1}}{q}\right)_{x}$

$$
+4 \beta\left(\frac{p r_{1}}{q^{2}}\right)_{x}+2 \beta \frac{1}{q}\left(\frac{p r_{2}}{q^{2}}\right)_{x}
$$$$
+2 \beta \frac{r_{2}}{q^{2}}\left(\frac{p}{q}\right)_{x}-4 \beta\left(\frac{p^{2} r_{2}}{q^{3}}\right)_{x} \text {, }
$$

$f_{1}=-2 \alpha\left(\frac{p}{q}\right)^{2}-2 \alpha \frac{1}{q}\left(\frac{p}{q}\right)_{x}-2 \beta \frac{1}{q}\left(\frac{r_{1}}{q}\right)_{x}$

$-4 \beta\left(\frac{p r_{1}}{q^{2}}\right)_{x}+2 \beta \frac{1}{q}\left(\frac{p r_{2}}{q^{2}}\right)_{x}$

$+2 \beta \frac{r_{2}}{q^{2}}\left(\frac{p}{q}\right)_{x}+4 \beta\left(\frac{p^{2} r_{2}}{q^{3}}\right)_{x}$,

$g_{0}=0$,

$h_{0}=\iota$,

$k_{0}=-l$,

$$
\begin{aligned}
g_{1}= & -2 \iota \frac{p}{q}-2 \beta \delta \frac{r_{1}}{q}-2 \alpha \frac{s_{1}}{q}+2 \alpha \delta \frac{p r_{2}}{q^{2}}+2 \beta \delta \frac{r_{1} r_{2}}{q^{2}} \\
& -2 \beta \delta \frac{p r_{2} r_{2}}{q^{3}}+2 \alpha \frac{p s_{2}}{q^{2}},
\end{aligned}
$$

$$
\begin{aligned}
k_{1}= & -2 \iota \frac{1}{q}\left(\frac{p}{q}\right)_{x}-2 \beta \delta \frac{1}{q}\left(\frac{r_{1}}{q}\right)_{x}+2 \beta \delta \frac{1}{q}\left(\frac{r_{1} r_{2}}{q^{2}}\right)_{x} \\
& +2 \beta \delta \frac{r_{2}}{q^{2}}\left(\frac{r_{1}}{q}\right)_{x}-2 \beta \delta \frac{1}{q}\left(\frac{p r_{2} r_{2}}{q^{3}}\right)_{x}
\end{aligned}
$$




$$
\begin{aligned}
& -2 \beta \delta \frac{r_{2}}{q^{2}}\left(\frac{p r_{2}}{q^{2}}\right)_{x}-2 \beta \delta \frac{r_{2} r_{2}}{q^{3}}\left(\frac{p}{q}\right)_{x} \\
& -2 \alpha \frac{1}{q}\left(\frac{s_{1}}{q}\right)_{x}+2 \alpha \delta \frac{r_{2}}{q^{2}}\left(\frac{p}{q}\right)_{x} \\
& +2 \alpha \delta \frac{1}{q}\left(\frac{p r_{2}}{q^{2}}\right)_{x}+2 \alpha \frac{s_{2}}{q^{2}}\left(\frac{p}{q}\right)_{x} \\
& +2 \alpha \frac{1}{q}\left(\frac{p s_{2}}{q^{2}}\right)_{x}-2 \iota\left(\frac{p}{q}\right)^{2}-4 \beta \delta \frac{p r_{1}}{q^{2}} \\
& +4 \beta \delta \frac{p r_{1} r_{2}}{q^{3}}+4 \beta \delta \frac{p r_{1} r_{2}}{q^{3}}-6 \beta \delta\left(\frac{p r_{2}}{q^{2}}\right)^{2} \\
& -2 \beta \delta\left(\frac{r_{1}}{q}\right)^{2}+4 \alpha \delta \frac{p^{2} r_{2}}{q^{3}}-4 \alpha \frac{p s_{1}}{q^{2}} \\
& +4 \alpha \frac{p^{2} s_{2}}{q^{3}} \\
& h_{1}=-2 \iota \frac{1}{q}\left(\frac{p}{q}\right)_{x}-2 \beta \delta \frac{1}{q}\left(\frac{r_{1}}{q}\right)_{x}+2 \beta \delta \frac{1}{q}\left(\frac{r_{1} r_{2}}{q^{2}}\right)_{x} \\
& +2 \beta \delta \frac{r_{2}}{q^{2}}\left(\frac{r_{1}}{q}\right)_{x}-2 \beta \delta \frac{1}{q}\left(\frac{p r_{2} r_{2}}{q^{3}}\right)_{x} \\
& -2 \beta \delta \frac{r_{2}}{q^{2}}\left(\frac{p r_{2}}{q^{2}}\right)_{x}-2 \beta \delta \frac{r_{2} r_{2}}{q^{3}}\left(\frac{p}{q}\right)_{x}
\end{aligned}
$$$$
-2 \alpha \frac{1}{q}\left(\frac{s_{1}}{q}\right)_{x}+2 \alpha \delta \frac{r_{2}}{q^{2}}\left(\frac{p}{q}\right)_{x}
$$$$
+2 \alpha \delta \frac{1}{q}\left(\frac{p r_{2}}{q^{2}}\right)_{x}+2 \alpha \frac{s_{2}}{q^{2}}\left(\frac{p}{q}\right)_{x}
$$$$
+2 \alpha \frac{1}{q}\left(\frac{p s_{2}}{q^{2}}\right)_{x}+2 \iota\left(\frac{p}{q}\right)^{2}+4 \beta \delta \frac{p r_{1}}{q^{2}}
$$$$
-4 \beta \delta \frac{p r_{1} r_{2}}{q^{3}}-4 \beta \delta \frac{p r_{1} r_{2}}{q^{3}}+6 \beta \delta\left(\frac{p r_{2}}{q^{2}}\right)^{2}
$$$$
+2 \beta \delta\left(\frac{r_{1}}{q}\right)^{2}-4 \alpha \delta \frac{p^{2} r_{2}}{q^{3}}+4 \alpha \frac{p s_{1}}{q^{2}}
$$$$
-4 \alpha \frac{p^{2} s_{2}}{q^{3}} \text {. }
$$

In order to get the integrable couplings systems from the enlarged zero curvature equations

$$
\bar{U}_{t_{m}}-V_{x}^{[m]}+\left[U, V^{[m]}\right]=0
$$

we define the Lax matrices and modification terms as

$$
V^{[m]}=\lambda\left(\lambda^{2 m+1} W\right)_{+}+\Delta_{m}, \quad m \geq 0
$$

where

$$
\begin{aligned}
& \Delta_{m}=\left(\begin{array}{ccc}
\Delta_{1 m} & \Delta_{2 m} & \Delta_{3 m} \\
0 & \Delta_{1 m} & \delta \Delta_{2 m} \\
0 & 0 & \Delta_{1 m}
\end{array}\right), \\
& \Delta_{1 m}=\left(\begin{array}{cc}
-a_{m+1} & \lambda \frac{q}{p} a_{m+1} \\
-\lambda \frac{q}{p} a_{m+1} & a_{m+1}
\end{array}\right), \\
& \Delta_{2 m}=\left(\begin{array}{cc}
-d_{m+1}-\frac{2 a_{1+m} r_{1}}{p} & \lambda \frac{q}{p} d_{m+1}+\lambda \frac{r_{2}}{p} a_{1+m} \\
-\lambda \frac{q}{p} d_{m+1}-\lambda \frac{r_{2}}{p} a_{1+m} & d_{m+1}+\frac{2 a_{1+m} r_{1}}{p}
\end{array}\right), \\
& \Delta_{1 m}=\left(\begin{array}{cc}
-g_{m+1}-2 \delta \frac{r_{1}}{p} d_{m+1}-\frac{2 s_{1}}{p} a_{1+m} & \lambda \frac{q}{p} g_{m+1}+\delta \lambda \frac{r_{2}}{p} d_{m+1}+\lambda \frac{s_{2}}{p} a_{1+m} \\
-\lambda \frac{q}{p} g_{m+1}-\delta \lambda \frac{r_{2}}{p} d_{m+1}-\lambda \frac{s_{2}}{p} a_{1+m} \quad g_{m+1}+2 \delta \frac{r_{1}}{p} d_{m+1}+\frac{2 s_{1}}{p} a_{1+m}
\end{array}\right),
\end{aligned}
$$

and $P_{+}$denotes the polynomial part of $P$. 
In this condition, the integrable couplings system can be obtained as

$$
u_{t_{m}}=\left(\begin{array}{c}
p_{t_{m}} \\
q_{t_{m}} \\
r_{1 t_{m}} \\
r_{2 t_{m}} \\
s_{1 t_{m}} \\
s_{2 t_{m}}
\end{array}\right)
$$

$$
=\left(\begin{array}{c}
\partial a_{m+1} \\
2 \partial \frac{q}{p} a_{m+1} \\
\partial d_{m+1}+2 \partial \frac{a_{1+m} r_{1}}{p} \\
2 \partial \frac{q}{p} d_{m+1}+2 \partial \frac{r_{2}}{p} a_{1+m} \\
\partial g_{m+1}+2 \delta \partial \frac{r_{1}}{p} d_{m+1}+2 \partial \frac{s_{1}}{p} a_{1+m} \\
2 \partial \frac{q}{p} g_{m+1}+2 \delta \partial \frac{r_{2}}{p} d_{m+1}+2 \partial \frac{s_{2}}{p} a_{1+m}
\end{array}\right)
$$

which is equal to

$$
u_{t_{m}}=\bar{J}\left(\begin{array}{c}
-4 a_{m+1} \eta_{1}-4 d_{m+1} \eta_{2}-4 g_{m+1} \eta_{3} \\
-b_{m+1} \eta_{1}+c_{m+1} \eta_{1}-e_{m+1} \eta_{2}+f_{m+1} \eta_{2}-h_{m+1} \eta_{3}+k_{m+1} \eta_{3} \\
-4 a_{m+1} \eta_{2}-4 d_{m+1} \delta \eta_{3} \\
-b_{m+1} \eta_{2}+c_{m+1} \eta_{2}-e_{m+1} \delta \eta_{3}+f_{m+1} \delta \eta_{3} \\
-4 a_{m+1} \eta_{3} \\
-b_{m+1} \eta_{3}+c_{m+1} \eta_{3}
\end{array}\right) \text {, }
$$

where

$$
\begin{aligned}
\bar{J} & =\left(\begin{array}{ccc}
0 & 0 & J_{1} \\
0 & \frac{1}{\delta} J_{1} & J_{2} \\
J_{1} & J_{2} & J_{3}
\end{array}\right), \\
J_{1} & =\left(\begin{array}{ll}
j_{1}^{1} & 0 \\
0 & j_{1}^{4}
\end{array}\right), \\
J_{2} & =\left(\begin{array}{cc}
j_{2}^{1} & j_{2}^{2} \\
j_{2}^{3} & j_{2}^{4}
\end{array}\right), \\
J_{3} & =\left(\begin{array}{cc}
j_{3}^{1} & j_{3}^{2} \\
j_{3}^{3} & j_{3}^{4}
\end{array}\right), \\
j_{1}^{1} & =-\frac{1}{4 \eta_{3}} \partial, \\
j_{1}^{4} & =\frac{1}{2 \eta_{3}} \partial \frac{q}{p} \partial^{-1} \frac{q}{p} \partial, \\
j_{2}^{1} & =\frac{\eta_{2}}{4 \delta \eta_{3} \eta_{3}} \partial, \\
j_{2}^{4} & =\frac{1}{2 \eta_{3}} \partial \frac{q}{p} \partial^{-1} \frac{r_{2}}{p} \partial+\frac{1}{2 \eta_{3}} \partial \frac{r_{2}}{p} \partial^{-1} \frac{q}{p} \partial \\
j_{2}^{3} & =\frac{1}{2 \eta_{3}} \partial \frac{r_{1}}{p} \partial^{-1} \frac{q}{p} \partial, \\
j_{2} & \frac{r_{1}}{p} \partial,
\end{aligned}
$$

$$
-\frac{\eta_{2}}{2 \delta \eta_{3} \eta_{3}} \partial \frac{q}{p} \partial^{-1} \frac{q}{p} \partial
$$$$
j_{3}^{1}=\frac{1}{2 \eta_{3}} \delta \partial \frac{r_{1}}{p} \partial^{-1} \frac{r_{1}}{p} \partial+\frac{\eta_{1}}{4 \eta_{3} \eta_{3}} \partial-\frac{\eta_{2} \eta_{2}}{4 \delta \eta_{3} \eta_{3} \eta_{3}} \partial \text {, }
$$$$
j_{3}^{2}=\frac{1}{2 \eta_{3}} \delta \partial \frac{r_{1}}{p} \partial^{-1} \frac{r_{2}}{p} \partial+\frac{1}{2 \eta_{3}} \partial \frac{s_{1}}{p} \partial^{-1} \frac{q}{p} \partial
$$$$
-\frac{\eta_{2}}{2 \eta_{3} \eta_{3}} \partial \frac{r_{1}}{p} \partial^{-1} \frac{q}{p} \partial
$$$$
j_{3}^{3}=\frac{1}{2 \eta_{3}} \delta \partial \frac{r_{2}}{p} \partial^{-1} \frac{r_{1}}{p} \partial+\frac{1}{2 \eta_{3}} \partial \frac{q}{p} \partial^{-1} \frac{s_{1}}{p} \partial
$$$$
-\frac{\eta_{2}}{2 \eta_{3} \eta_{3}} \partial \frac{q}{p} \partial^{-1} \frac{r_{1}}{p} \partial
$$$$
j_{3}^{4}=\frac{1}{2 \eta_{3}} \partial \frac{q}{p} \partial^{-1} \frac{s_{2}}{p} \partial+\frac{1}{2 \eta_{3}} \partial \frac{s_{2}}{p} \partial^{-1} \frac{q}{p} \partial
$$$$
+\frac{1}{2 \eta_{3}} \delta \partial \frac{r_{2}}{p} \partial^{-1} \frac{r_{2}}{p} \partial-\frac{\eta_{1}}{2 \eta_{3} \eta_{3}} \partial \frac{q}{p} \partial^{-1} \frac{q}{p} \partial
$$$$
-\frac{\eta_{2}}{2 \eta_{3} \eta_{3}} \partial \frac{q}{p} \partial^{-1} \frac{r_{2}}{p} \partial-\frac{\eta_{2}}{2 \eta_{3} \eta_{3}} \partial \frac{r_{2}}{p} \partial^{-1} \frac{q}{p} \partial
$$$$
+\frac{\eta_{2} \eta_{2}}{2 \delta \eta_{3} \eta_{3} \eta_{3}} \partial \frac{q}{p} \partial^{-1} \frac{q}{p} \partial \text {. }
$$

Remark 4. $\bar{U}$ and $V^{[m]}$ are the Lax pairs of the integrable couplings of the 2-CQLTH; this implies that the integrable couplings are integrable in Lax sense. 


\section{Bi-Hamiltonian Structures and Liouville Integrability of the Bi-Integrable Couplings}

Because the associated matrix Lie algebras are nonsemisimple, we should use the variational identity (11) to furnish the Hamiltonian structures of the integrable couplings system (58) (or (59)). Before doing this, we should find out the nondegenerate, symmetric, and adinvariant bilinear form corresponding to the nonsemisimple matrix loop algebras first [22].

By defining a mapping from $\bar{g}$ to $R^{9}$ as

$$
\begin{aligned}
\sigma: \bar{g} & \longrightarrow R^{9}, \\
A & \longmapsto\left(a_{1}, \ldots, a_{9}\right)^{T},
\end{aligned}
$$

where

$$
\begin{aligned}
A & =M\left(A_{1}, A_{2}, A_{3}\right) \in \bar{g}, \\
A_{i} & =\left(\begin{array}{cc}
a_{3 i-2} & a_{3 i-1} \\
a_{3 i} & -a_{3 i-2}
\end{array}\right),
\end{aligned}
$$

$$
i=1,2,3 \text {, }
$$

we have the following results according to [22, 28, 30].

The mapping (61) is a Lie algebra isomorphism from $\bar{g}$ to $R^{9}$ and the Lie bracket $[\cdot, \cdot]$ can be calculated as

$$
\begin{aligned}
{[a, b] } & =a^{T} R(b), \\
a & =\left(a_{1}, a_{2}, \ldots, a_{9}\right), \\
b & =\left(b_{1}, b_{2}, \ldots, b_{9}\right) \in R^{9},
\end{aligned}
$$

where

$$
\begin{aligned}
R(b) & =M\left(R_{1}, R_{2}, R_{3}\right), \\
R & =\left(\begin{array}{ccc}
0 & 2 b_{3 i-1} & -2 b_{3 i} \\
b_{3 i} & -2 b_{3 i-2} & 0 \\
-b_{3 i-1} & 0 & 2 b_{3 i-2}
\end{array}\right), \quad i=1,2,3 .
\end{aligned}
$$

Assuming that the bilinear form on $R^{9}$ possesses the following form:

$$
\langle a, b\rangle=a^{T} F b,
$$

where $F$ is a constant matrix, the symmetric property and adinvariance property of $F$

$$
\begin{aligned}
\langle a, b\rangle & =\langle b, a\rangle, \\
\langle a,[b, c]\rangle & =\langle[a, b], c\rangle
\end{aligned}
$$

give rise to

$$
\begin{aligned}
F & =F^{T}, \\
F(R(b))^{T} & =-R(b) F, \quad b \in R^{9} .
\end{aligned}
$$

So the form of the constant matrix $F$ can be written as

$$
F=\left(\begin{array}{ccc}
\eta_{1} & \eta_{2} & \eta_{3} \\
\eta_{2} & \delta \eta_{3} & 0 \\
\eta_{3} & 0 & 0
\end{array}\right) \otimes\left(\begin{array}{lll}
2 & 0 & 0 \\
0 & 0 & 1 \\
0 & 1 & 0
\end{array}\right)
$$

where $\eta_{1}, \eta_{2}$, and $\eta_{3}$ are arbitrary constants. Thus, the bilinear form on the semidirect sums $\bar{g}$ of $g$ and $g_{c}$ can be defined as

$$
\begin{aligned}
\langle A, B\rangle & =\langle\sigma(A), \sigma(B)\rangle_{R^{9}} \\
& =\left(a_{1}, a_{2}, \ldots, a_{9}\right) F\left(b_{1}, b_{2}, \ldots, b_{9}\right)^{T},
\end{aligned}
$$

where $A=\sigma^{-1}\left(\left(a_{1}, a_{2}, \ldots, a_{9}\right)^{T}\right), B=\sigma^{-1}\left(\left(b_{1}, b_{2}, \ldots, b_{9}\right)^{T}\right) \epsilon$ $\bar{g}$.

In order to guarantee that the bilinear form is nondegenerate, we should require

$$
\operatorname{det}(F)=8 \delta^{3} \eta_{3}^{9} \neq 0
$$

that is to say, $\delta \neq 0$ and $\eta_{3} \neq 0$.

By calculation, we have

$$
\begin{aligned}
\frac{\partial \bar{U}}{\partial \lambda} & =\left(\begin{array}{cccccc}
0 & \frac{q}{2} & 0 & \frac{r_{2}}{2} & 0 & \frac{s_{2}}{2} \\
-\frac{q}{2} & 0 & -\frac{r_{2}}{2} & 0 & -\frac{s_{2}}{2} & 0 \\
0 & 0 & 0 & \frac{q}{2} & 0 & \delta \frac{r_{2}}{2} \\
0 & 0 & -\frac{q}{2} & 0 & -\delta \frac{r_{2}}{2} & 0 \\
0 & 0 & 0 & 0 & 0 & \frac{q}{2} \\
0 & 0 & 0 & 0 & -\frac{q}{2} & 0
\end{array}\right), \\
\frac{\partial \bar{U}}{\partial p} & =\left(\begin{array}{cccccc}
-1 & 0 & 0 & 0 & 0 & 0 \\
0 & 1 & 0 & 0 & 0 & 0 \\
0 & 0 & -1 & 0 & 0 & 0 \\
0 & 0 & 0 & 1 & 0 & 0 \\
0 & 0 & 0 & 0 & -1 & 0 \\
0 & 0 & 0 & 0 & 0 & 1
\end{array}\right)
\end{aligned}
$$

$$
\frac{\partial \bar{U}}{\partial q}=\left(\begin{array}{cccccc}
0 & \frac{\lambda}{2} & 0 & 0 & 0 & 0 \\
-\frac{\lambda}{2} & 0 & 0 & 0 & 0 & 0 \\
0 & 0 & 0 & \frac{\lambda}{2} & 0 & 0 \\
0 & 0 & -\frac{\lambda}{2} & 0 & 0 & 0 \\
0 & 0 & 0 & 0 & 0 & \frac{\lambda}{2} \\
0 & 0 & 0 & 0 & -\frac{\lambda}{2} & 0
\end{array}\right),
$$




$$
\begin{aligned}
& \frac{\partial \bar{U}}{\partial r_{1}}=\left(\begin{array}{cccccc}
0 & 0 & -1 & 0 & 0 & 0 \\
0 & 0 & 0 & 1 & 0 & 0 \\
0 & 0 & 0 & 0 & -\delta & 0 \\
0 & 0 & 0 & 0 & 0 & \delta \\
0 & 0 & 0 & 0 & 0 & 0 \\
0 & 0 & 0 & 0 & 0 & 0
\end{array}\right) \\
& \frac{\partial \bar{U}}{\partial r_{2}}=\left(\begin{array}{cccccc}
0 & 0 & 0 & \frac{\lambda}{2} & 0 & 0 \\
0 & 0 & -\frac{\lambda}{2} & 0 & 0 & 0 \\
0 & 0 & 0 & 0 & 0 & \delta \frac{\lambda}{2} \\
0 & 0 & 0 & 0 & -\delta \frac{\lambda}{2} & 0 \\
0 & 0 & 0 & 0 & 0 & 0 \\
0 & 0 & 0 & 0 & 0 & 0
\end{array}\right)
\end{aligned}
$$

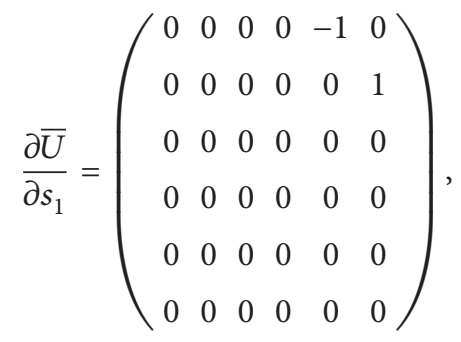

$\frac{\partial \bar{U}}{\partial s_{2}}=\left(\begin{array}{cccccc}0 & 0 & 0 & 0 & 0 & \frac{\lambda}{2} \\ 0 & 0 & 0 & 0 & -\frac{\lambda}{2} & 0 \\ 0 & 0 & 0 & 0 & 0 & 0 \\ 0 & 0 & 0 & 0 & 0 & 0 \\ 0 & 0 & 0 & 0 & 0 & 0 \\ 0 & 0 & 0 & 0 & 0 & 0\end{array}\right)$

Further, we have

$\left\langle W \frac{\partial U}{\partial \lambda}\right\rangle=-\frac{1}{2} b q \eta_{1}+\frac{1}{2} c q \eta_{1}-\frac{1}{2} e q \eta_{2}+\frac{1}{2} f q \eta_{2}-\frac{1}{2} b r_{2} \eta_{2}+\frac{1}{2} c r_{2} \eta_{2}-\frac{1}{2} h q \eta_{3}+\frac{1}{2} k q \eta_{3}-\frac{1}{2} e \delta r_{2} \eta_{3}+\frac{1}{2} f \delta r_{2} \eta_{3}-\frac{1}{2} b s_{2} \eta_{3}$ $+\frac{1}{2} c s_{2} \eta_{3}$

$$
\left\langle W \frac{\partial U}{\partial u}\right\rangle=\left(\begin{array}{c}
-2 a \eta_{1}-2 d \eta_{2}-2 g \eta_{3} \\
-\frac{1}{2} b \lambda \eta_{1}+\frac{1}{2} c \lambda \eta_{1}-\frac{1}{2} e \lambda \eta_{2}+\frac{1}{2} f \lambda \eta_{2}-\frac{1}{2} h \lambda \eta_{3}+\frac{1}{2} k \lambda \eta_{3} \\
-2 a \eta_{2}-2 d \delta \eta_{3} \\
-\frac{1}{2} b \lambda \eta_{2}+\frac{1}{2} c \lambda \eta_{2}-\frac{1}{2} e \delta \lambda \eta_{3}+\frac{1}{2} f \delta \lambda \eta_{3} \\
-2 a \eta_{3} \\
-\frac{1}{2} b \lambda \eta_{3}+\frac{1}{2} c \lambda \eta_{3}
\end{array}\right) .
$$

Substituting the above expressions into the variational identity (11), we can obtain

$$
\left.\begin{array}{c}
\frac{\delta}{\delta \bar{u}} \int\left(-\frac{1}{2} b q \eta_{1}+\frac{1}{2} c q \eta_{1}-\frac{1}{2} e q \eta_{2}+\frac{1}{2} f q \eta_{2}-\frac{1}{2} b r_{2} \eta_{2}+\frac{1}{2} c r_{2} \eta_{2}-\frac{1}{2} h q \eta_{3}+\frac{1}{2} k q \eta_{3}-\frac{1}{2} e \delta r_{2} \eta_{3}+\frac{1}{2} f \delta r_{2} \eta_{3}-\frac{1}{2} b s_{2} \eta_{3}\right. \\
-2 a \eta_{1}-2 d \eta_{2}-2 g \eta_{3} \\
\left.+\frac{1}{2} c s_{2} \eta_{3}\right) d x=\lambda^{-\gamma} \frac{\partial}{\partial \lambda} \lambda^{\gamma}\left(\begin{array}{c}
-\frac{1}{2} b \lambda \eta_{1}+\frac{1}{2} c \lambda \eta_{1}-\frac{1}{2} e \lambda \eta_{2}+\frac{1}{2} f \lambda \eta_{2}-\frac{1}{2} h \lambda \eta_{3}+\frac{1}{2} k \lambda \eta_{3} \\
-2 a \eta_{2}-2 d \delta \eta_{3} \\
-\frac{1}{2} b \lambda \eta_{2}+\frac{1}{2} c \lambda \eta_{2}-\frac{1}{2} e \delta \lambda \eta_{3}+\frac{1}{2} f \delta \lambda \eta_{3} \\
-2 a \eta_{3} \\
-\frac{1}{2} b \lambda \eta_{3}+\frac{1}{2} c \lambda \eta_{3}
\end{array}\right) .
\end{array}\right) .
$$


Comparing the coefficients of all powers of $\lambda$ in (73), we know that

$$
\frac{\delta}{\delta \bar{u}} \int \frac{z_{m}}{1-2 m} d x
$$

$$
=\left(\begin{array}{c}
-4 a_{m} \eta_{1}-4 d_{m} \eta_{2}-4 g_{m} \eta_{3} \\
-b_{m} \eta_{1}+c_{m} \eta_{1}-e_{m} \eta_{2}+f_{m} \eta_{2}-h_{m} \eta_{3}+k_{m} \eta_{3} \\
-4 a_{m} \eta_{2}-4 d_{m} \delta \eta_{3} \\
-b_{m} \eta_{2}+c_{m} \eta_{2}-e_{m} \delta \eta_{3}+f_{m} \delta \eta_{3} \\
-4 a_{m} \eta_{3} \\
-b_{m} \eta_{3}+c_{m} \eta_{3}
\end{array}\right),
$$

$m \geq 0$,

where

$$
\begin{aligned}
z_{m}= & -b_{m} q \eta_{1}+c_{m} q \eta_{1}-e_{m} q \eta_{2}+f_{m} q \eta_{2}-b_{m} r_{2} \eta_{2} \\
& +c_{m} r_{2} \eta_{2}-h_{m} q \eta_{3}+k_{m} q \eta_{3}-e_{m} \delta r_{2} \eta_{3} \\
& +f_{m} \delta r_{2} \eta_{3}-b_{m} s_{2} \eta_{3}+c_{m} s_{2} \eta_{3} .
\end{aligned}
$$

Setting $m=1$ in (74), we know that the constant $\gamma=1$, so we have

$$
\begin{gathered}
\frac{\delta \mathscr{H}_{m}}{\delta u} \\
=\left(\begin{array}{c}
-4 a_{m} \eta_{1}-4 d_{m} \eta_{2}-4 g_{m} \eta_{3} \\
-b_{m} \eta_{1}+c_{m} \eta_{1}-e_{m} \eta_{2}+f_{m} \eta_{2}-h_{m} \eta_{3}+k_{m} \eta_{3} \\
-4 a_{m} \eta_{2}-4 d_{m} \delta \eta_{3} \\
-b_{m} \eta_{2}+c_{m} \eta_{2}-e_{m} \delta \eta_{3}+f_{m} \delta \eta_{3} \\
-4 a_{m} \eta_{3} \\
-b_{m} \eta_{3}+c_{m} \eta_{3}
\end{array}\right) .
\end{gathered}
$$

Thus, the Hamiltonian functions can be expressed as

$$
\mathscr{H}_{m}=\int \frac{z_{m}}{1-2 m} d x
$$

and the Hamiltonian structures of (58) as

$$
u_{t_{m}}=J \frac{\delta \mathscr{H}_{m+1}}{\delta u}, \quad m \geq 0 .
$$

Furthermore, by using (52), we can get

$$
u_{t_{m}}=J \frac{\delta \mathscr{H}_{m+1}}{\delta u}=M \frac{\delta \mathscr{H}_{m}}{\delta u},
$$

where

$$
\begin{aligned}
\bar{M} & =\left(\begin{array}{ccc}
0 & 0 & \bar{J}_{1} \\
0 & \frac{1}{\delta} \bar{J}_{1} & \bar{J}_{2} \\
\bar{J}_{1} & \bar{J}_{2} & \bar{J}_{3}
\end{array}\right), \\
\bar{J}_{1} & =\left(\begin{array}{cc}
\bar{j}_{1}^{1} & 0 \\
0 & \bar{j}_{1}
\end{array}\right), \\
\bar{J}_{2} & =\left(\begin{array}{ll}
\bar{j}_{2} & \bar{j}_{2}^{2} \\
\bar{j}_{2}^{3} & \bar{j}_{2}^{4}
\end{array}\right),
\end{aligned}
$$

$$
\bar{J}_{3}=\left(\begin{array}{cc}
\bar{j}_{3} & \bar{j}_{3}^{2} \\
\bar{j}_{3} & \bar{j}_{3}^{4}
\end{array}\right)
$$$$
\bar{j}_{1}^{1}=\frac{1}{4 \eta_{3}} \partial \frac{1}{q} \partial \frac{1}{q} \partial-\frac{1}{\eta_{3}} \partial \frac{p}{q} \partial^{-1} \frac{p}{q} \partial,
$$$$
\bar{j}_{1}=2 \frac{1}{\eta_{3}} \partial-\frac{1}{2 \eta_{3}} \partial \frac{1}{p} \partial \frac{1}{p} \partial \text {, }
$$$$
\bar{j}_{2}^{1}=-\frac{1}{4 \eta_{3}} \partial \frac{r_{2}}{q^{2}} \partial \frac{1}{q} \partial-\frac{1}{4 \eta_{3}} \partial \frac{1}{q} \partial \frac{r_{2}}{q^{2}} \partial+\frac{1}{\eta_{3}} \partial \frac{p}{q} \partial^{-1} \frac{p r_{2}}{q^{2}} \partial
$$$$
+\frac{1}{\eta_{3}} \partial \frac{p r_{2}}{q^{2}} \partial^{-1} \frac{p}{q} \partial-\frac{1}{\eta_{3}} \partial \frac{p}{q} \partial^{-1} \frac{r_{1}}{q} \partial-\frac{1}{\eta_{3}} \partial \frac{r_{1}}{q} \partial^{-1} \frac{p}{q} \partial
$$$$
+\frac{\eta_{2}}{\delta \eta_{3} \eta_{3}} \partial \frac{p}{q} \partial^{-1} \frac{p}{q} \partial-\frac{\eta_{2}}{4 \delta \eta_{3} \eta_{3}} \partial \frac{1}{q} \partial \frac{1}{q} \partial,
$$$$
\bar{j}_{2}^{2}=-\frac{1}{2 \eta_{3}} \partial \frac{r_{1}}{p q} \partial \frac{1}{p} \partial+2 \frac{1}{\eta_{3}} \partial \frac{r_{1}}{q},
$$$$
\bar{j}_{2}^{3}=-\frac{1}{2 \eta_{3}} \partial \frac{1}{p} \partial \frac{r_{1}}{p q} \partial+2 \frac{1}{\eta_{3}} \frac{r_{1}}{q} \partial,
$$$$
\hat{j}_{2}=2 \frac{1}{\eta_{3}} \partial \frac{r_{1}}{p}+2 \frac{1}{\eta_{3}} \frac{r_{1}}{p} \partial-2 \frac{\eta_{2}}{\delta \eta_{3} \eta_{3}} \partial
$$

$$
+\frac{\eta_{2}}{2 \delta \eta_{3} \eta_{3}} \partial \frac{1}{p} \partial \frac{1}{p} \partial
$$

$$
\begin{aligned}
\bar{j}_{3} & =-\frac{1}{4 \eta_{3}}\left(4 \partial \frac{p}{q} \partial^{-1} \frac{s_{1}}{q} \partial+4 \partial \frac{s_{1}}{q} \partial^{-1} \frac{p}{q} \partial-\delta \partial \frac{1}{q} \partial \frac{r_{2} r_{2}}{q^{3}} \partial\right. \\
& -\delta \partial \frac{r_{2} r_{2}}{q^{3}} \partial \frac{1}{q} \partial+\partial \frac{1}{q} \partial \frac{s_{2}}{q^{2}} \partial+\partial \frac{s_{2}}{q^{2}} \partial \frac{1}{q} \partial \\
& -4 \delta \partial \frac{r_{1}}{q} \partial^{-1} \frac{p r_{2}}{q^{2}} \partial-4 \delta \partial \frac{p r_{2}}{q^{2}} \partial^{-1} \frac{r_{1}}{q} \partial \\
& +4 \delta \partial \frac{r_{1}}{q} \partial^{-1} \frac{r_{1}}{q} \partial-\delta \partial \frac{r_{2}}{q^{2}} \partial \frac{r_{2}}{q^{2}} \partial+4 \delta \partial \frac{p r_{2}}{q^{2}} \partial^{-1} \frac{p r_{2}}{q^{2}} \partial \\
& +4 \delta \partial \frac{p r_{2} r_{2}}{q^{3}} \partial^{-1} \frac{p}{q} \partial+4 \delta \partial \frac{p}{q} \partial^{-1} \frac{p r_{2} r_{2}}{q^{3}} \partial \\
& -4 \partial \frac{p s_{2}}{q^{2}} \partial^{-1} \frac{p}{q} \partial-4 \partial \frac{p}{q} \partial^{-1} \frac{p s_{2}}{q^{2}} \partial-4 \delta \partial \frac{p}{q} \partial^{-1} \frac{r_{1} r_{2}}{q^{2}} \partial
\end{aligned}
$$




$$
\begin{aligned}
& -4 \delta \partial \frac{r_{1} r_{2}}{q^{2}} \partial^{-1} \frac{p}{q} \partial+\frac{\eta_{1}}{\eta_{3}} \partial \frac{1}{q} \partial \frac{1}{q} \partial-\frac{\eta_{2} \eta_{2}}{\delta \eta_{3} \eta_{3}} \partial \frac{1}{q} \partial \frac{1}{q} \partial \\
& -4 \frac{\eta_{1}}{\eta_{3}} \partial \frac{p}{q} \partial^{-1} \frac{p}{q} \partial+4 \frac{\eta_{2} \eta_{2}}{\delta \eta_{3} \eta_{3}} \partial \frac{p}{q} \partial^{-1} \frac{p}{q} \partial \\
& -\frac{\eta_{2}}{\eta_{3}} \partial \frac{r_{2}}{q^{2}} \partial \frac{1}{q} \partial-\frac{\eta_{2}}{\eta_{3}} \partial \frac{1}{q} \partial \frac{r_{2}}{q^{2}} \partial-4 \frac{\eta_{2}}{\eta_{3}} \partial \frac{p}{q} \partial^{-1} \frac{r_{1}}{q} \partial \\
& -4 \frac{\eta_{2}}{\eta_{3}} \partial \frac{r_{1}}{q} \partial^{-1} \frac{p}{q} \partial+2 \delta \partial \frac{r_{1}}{p q} \partial \frac{r_{1}}{p q} \partial \\
& \left.+4 \frac{\eta_{2}}{\eta_{3}} \partial \frac{p}{q} \partial^{-1} \frac{p r_{2}}{q^{2}} \partial+4 \frac{\eta_{2}}{\eta_{3}} \partial \frac{p r_{2}}{q^{2}} \partial^{-1} \frac{p}{q} \partial\right), \\
& \bar{j}_{3}^{2}=\frac{1}{2 \eta_{3}} \delta \partial \frac{r_{1} r_{2}}{p q^{2}} \partial \frac{1}{p} \partial-2 \frac{1}{\eta_{3}} \delta \partial \frac{r_{1} r_{2}}{q^{2}}+2 \frac{1}{\eta_{3}} \delta \partial \frac{r_{1} r_{1}}{p q} \\
& -\frac{1}{2 \eta_{3}} \partial \frac{s_{1}}{p q} \partial \frac{1}{p} \partial+2 \frac{1}{\eta_{3}} \partial \frac{s_{1}}{q}+\frac{1}{2} \frac{\eta_{2}}{\eta_{3} \eta_{3}} \partial \frac{r_{1}}{p q} \partial \frac{1}{p} \partial-2 \\
& \cdot \frac{\eta_{2}}{\eta_{3} \eta_{3}} \partial \frac{r_{1}}{q} \\
& \bar{j}_{3}^{3}=\frac{1}{2 \eta_{3}} \delta \partial \frac{1}{p} \partial \frac{r_{1} r_{2}}{p q^{2}} \partial-2 \frac{1}{\eta_{3}} \delta \frac{r_{1} r_{2}}{q^{2}} \partial+2 \frac{1}{\eta_{3}} \delta \frac{r_{1} r_{1}}{p q} \partial \\
& -\frac{1}{2 \eta_{3}} \partial \frac{1}{p} \partial \frac{s_{1}}{p q} \partial+2 \frac{1}{\eta_{3}} \frac{s_{1}}{q} \partial+\frac{1}{2} \frac{\eta_{2}}{\eta_{3} \eta_{3}} \partial \frac{1}{p} \partial \frac{r_{1}}{p q} \partial-2 \\
& \cdot \frac{\eta_{2}}{\eta_{3} \eta_{3}} \frac{r_{1}}{q} \partial \\
& \bar{j}_{3}^{4}=-2 \frac{1}{\eta_{3}} \partial \frac{s_{1}}{p}-2 \frac{1}{\eta_{3}} \frac{s_{1}}{p} \partial+\frac{\eta_{1}}{2 \eta_{3} \eta_{3}} \partial \frac{1}{p} \partial \frac{1}{p} \partial+2 \\
& \cdot \frac{\eta_{2}}{\eta_{3} \eta_{3}} \partial \frac{r_{1}}{p}+2 \frac{\eta_{2}}{\eta_{3} \eta_{3}} \frac{r_{1}}{p} \partial-\frac{\eta_{2} \eta_{2}}{2 \delta \eta_{3} \eta_{3} \eta_{3}} \partial \frac{1}{p} \partial \frac{1}{p} \partial-2 \\
& \cdot \frac{\eta_{2} \eta_{2}}{\delta \eta_{3} \eta_{3} \eta_{3}} \partial+2 \frac{\eta_{1}}{\eta_{3} \eta_{3}} \partial \text {. }
\end{aligned}
$$

By a complicated computation, we know that

$$
\begin{gathered}
\bar{J}=-\bar{J}^{*} \\
\bar{M}=-\bar{M}^{*} ;
\end{gathered}
$$

namely, the hierarchy (58) possess bi-Hamiltonian structures

$$
u_{t_{m}}=J \frac{\delta \mathscr{H}_{m+1}}{\delta u}=M \frac{\delta \mathscr{H}_{m}}{\delta u}, \quad m \geq 0,
$$

where $\bar{J}$ and $\bar{M}$ are the Hamiltonian pairs of the integrable couplings of the 2-CQLTH. So the integrable couplings (58) are Liouville integrable.

\section{Conclusion}

In this paper, we construct a new eigenvalue problem and give the two-component Casimir-Qiao-Liu type hierarchy (2-CQLTH). The 2-CQLTH has Lax representation and possesses bi-Hamiltonian structures, which implies that it is integrable in both Lax and Liouville sense. Furthermore, we obtain the integrable couplings of the integrable hierarchy as well as its Lax pairs and bi-Hamiltonian structures and prove that the integrable couplings are also integrable in both Lax and Liouville senses.

\section{Competing Interests}

The authors declare that they have no competing interests.

\section{Acknowledgments}

The author Yuqin Yao is grateful to Professor Wen-Xiu Ma for his stimulating discussions. This work is supported in part by the National Natural Science Foundation of China (Grants no. 11301179) and China Scholarship Council.

\section{References}

[1] P. J. Olver and P. Rosenau, "Tri-Hamiltonian duality between solitons and solitary-wave solutions having compact support," Physical Review E. Statistical, Nonlinear, and Soft Matter Physics, vol. 53, no. 2, pp. 1900-1906, 1996.

[2] Z. J. Qiao and L. P. Liu, "A new integrable equation with no smooth solitons," Chaos, Solitons \& Fractals, vol. 41, no. 2, pp. 587-593, 2009.

[3] Z. J. Qiao, "New integrable hierarchy, its parametric solutions, cuspons, one-peak solitons, and M/W-shape peak solitons," Journal of Mathematical Physics, vol. 48, no. 8, Article ID 082701, 20 pages, 2007.

[4] J. Li and Z. Qiao, "Bifurcations of traveling wave solutions for an integrable equation," Journal of Mathematical Physics, vol. 51, article 042703, 2010.

[5] V. Marinakis, "Higher-order equations of the KdV type are integrable," Advances in Mathematical Physics, vol. 2010, Article ID 329586, 5 pages, 2010.

[6] S. Sakovich, "Smooth soliton solutions of a new integrable equation by Qiao," Journal of Mathematical Physics, vol. 52, article 023509, 2011.

[7] W. X. Ma, "Integrable couplings of vector AKNS soliton equations," Journal of Mathematical Physics, vol. 46, no. 3, pp. 033507-033525, 2005.

[8] T.-C. Xia, F.-J. Yu, and D.-Y. Chen, "The multi-component Yang hierarchy and its multi-component integrable coupling system with two arbitrary functions," Chaos, Solitons and Fractals, vol. 24, no. 1, pp. 235-240, 2005.

[9] L. Luo and E. Fan, "The algebraic structure of discrete zero curvature equations associated with integrable couplings and application to enlarged Volterra systems," Science in China. Series A. Mathematics, vol. 52, no. 1, pp. 147-159, 2009.

[10] Y. Zhang and E. Fan, "Coupling integrable couplings and biHamiltonian structure associated with the Boiti-PempinelliTu hierarchy," Journal of Mathematical Physics, vol. 51, article 083506, 2010.

[11] G. Z. Tu, "The trace identity, a powerful tool for constructing the Hamiltonian structure of integrable systems," Journal of Mathematical Physics, vol. 30, no. 2, pp. 330-338, 1989.

[12] G. Z. Tu, "A trace identity and its applications to the theory of discrete integrable systems," Journal of Physics A: Mathematical and General, vol. 23, no. 17, pp. 3903-3922, 1990. 
[13] W. X. Ma and M. Chen, "Hamiltonian and quasi-Hamiltonian structures associated with semi-direct sums of Lie algebras," Journal of Physics A: General Physics, vol. 39, no. 34, pp. 1078710801, 2006.

[14] W.-X. Ma, X.-X. Xu, and Y. F. Zhang, "Semi-direct sums of Lie algebras and continuous integrable couplings," Physics Letters A, vol. 351, no. 3, pp. 125-130, 2006.

[15] W.-X. Ma, X.-X. Xu, and Y. F. Zhang, "Semidirect sums of Lie algebras and discrete integrable couplings," Journal of Mathematical Physics, vol. 47, no. 5, Article ID 053501, 5 pages, 2006.

[16] W. X. Ma, "A new hierarchy of Liouville integrable generalized Hamiltonian equations and its reduction," Chinese Annals of Mathematics, Series A, vol. 13, no. 1, pp. 115-123, 1992 (Chinese).

[17] Y. B. Zeng and X. Cao, "Separation of variables for higherorder binary constrained flows of the Tu hierarchy," Advance in Mathematics, vol. 2, no. 2, pp. 135-147, 2002.

[18] X. X. Xu, "A generalized Wadati-Konno-Ichikawa hierarchy and new finite-dimensional integrable systems," Physics Letters A, vol. 301, no. 3-4, pp. 250-262, 2002.

[19] W.-X. Ma, "Variational identities and applications to Hamiltonian structures of soliton equations," Nonlinear Analysis: Theory, Methods \& Applications, vol. 71, no. 12, pp. e1716-e1726, 2009.

[20] F. Guo and Y. Zhang, "The quadratic-form identity for constructing the Hamiltonian structure of integrable systems," Journal of Physics. A. Mathematical and General, vol. 38, no. 40, pp. 8537-8548, 2005.

[21] W.-X. Ma, "Integrable couplings of vector AKNS soliton equations," Journal of Mathematical Physics, vol. 46, no. 3, pp. 033507-033525, 2005.

[22] S. M. Yu, Y. Q. Yao, S. F. Shen, and W.-X. Ma, "Bi-integrable couplings of a Kaup-Newell type soliton hierarchy and their biHamiltonian structures," Communications in Nonlinear Science \& Numerical Simulation, vol. 23, no. 1-3, pp. 366-377, 2015.

[23] Y. Zhang, H. Dong, and Z. Li, "Integrable couplings of the TB hierarchy and its Hamiltonian structure," Annals of Differential Equations, vol. 24, no. 1, pp. 112-116, 2008.

[24] W. X. Ma, J. H. Meng, and H. Q. Zhang, "Integrable couplings, variationmal identities and Hamiltonian formulations," in Proceedings of the 6th International Federation of Nonlinear Analysts Conference, pp. 1-17, 2012.

[25] F. K. Guo and Y. F. Zhang, "The integrable coupling of the AKNS hierarchy and its Hamiltonian structure," Chaos, Solitons \& Fractals, vol. 32, no. 5, pp. 1898-1902, 2007.

[26] W. X. Ma, "A soliton hierarhy associated with so(3,R)," Applied Mathematics \& Computation, vol. 220, no. 4, pp. 117-122, 2013.

[27] W.-X. Ma, "A spectral problem based on $(3, \mathrm{R})$ and its associated commuting soliton equations," Journal of Mathematical Physics, vol. 54, no. 10, Article ID 103509, 2013.

[28] W. X. Ma, "Loop algebras and bi-integrable couplings," Chinese Annals of Mathematics, Series B, vol. 33, no. 2, pp. 207-224, 2012.

[29] W. X. Ma, "Integrable couplings and matrix loop algebra," in Proceedings of the Nonlinear and Modern Mathematical Physics: Proceedings of the 2nd International Workshop, vol. 1562 of AIP Conference Proceedings, pp. 105-122, Tampa, Fla, USA, March 2013.

[30] W.-X. Ma, "Nonlinear continuous integrable Hamiltonian couplings," Applied Mathematics \& Computation, vol. 217, no. 17, pp. 7238-7244, 2011. 


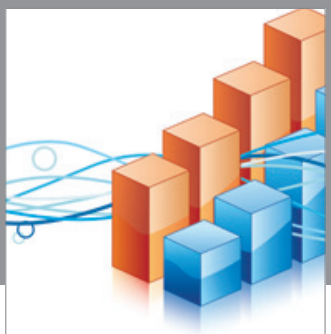

Advances in

Operations Research

vatem alat4

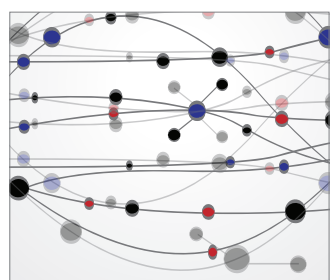

\section{The Scientific} World Journal
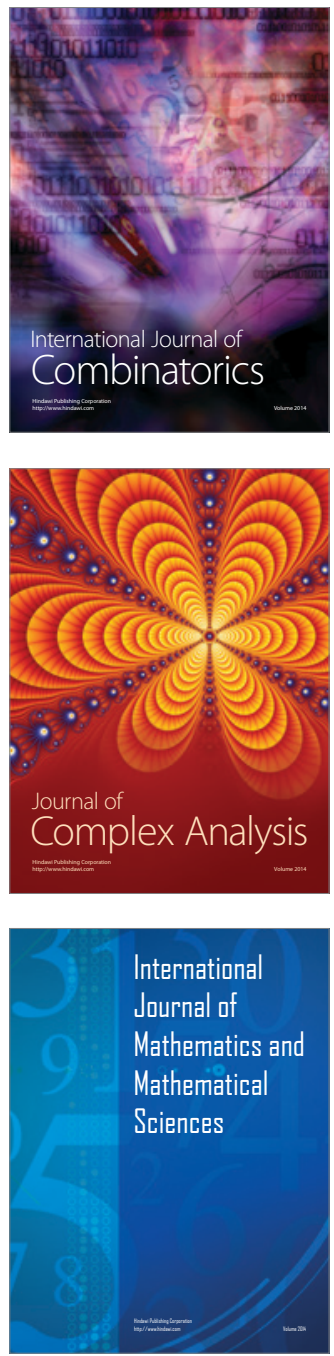
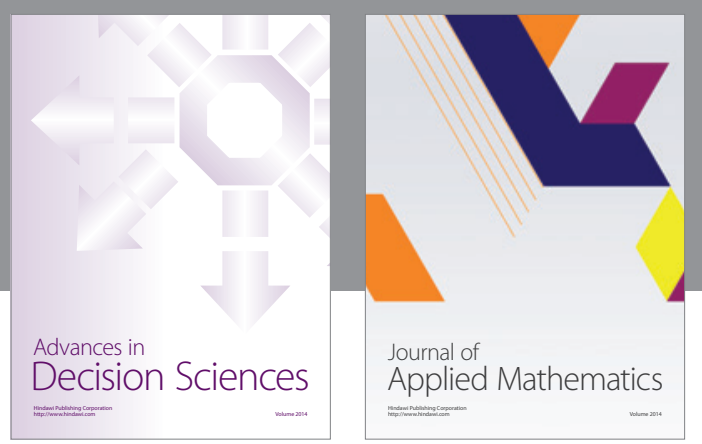

Algebra

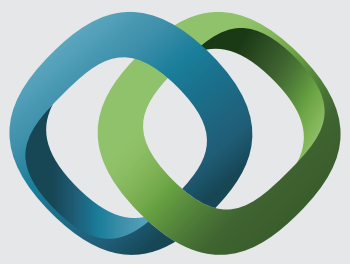

\section{Hindawi}

Submit your manuscripts at

http://www.hindawi.com
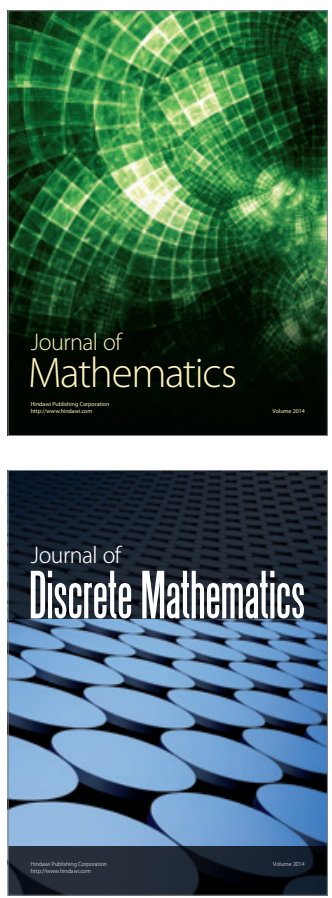

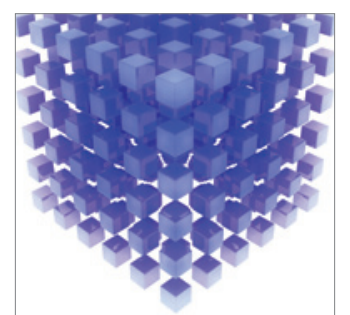

Mathematical Problems in Engineering
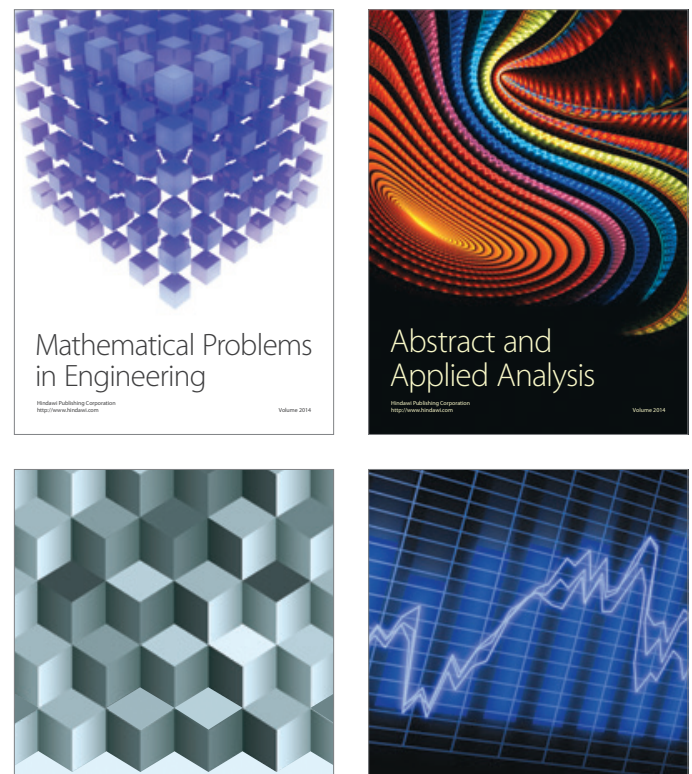

Journal of

Function Spaces

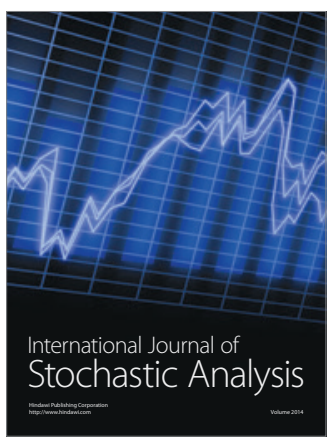

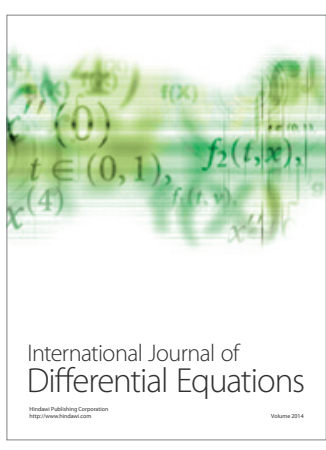
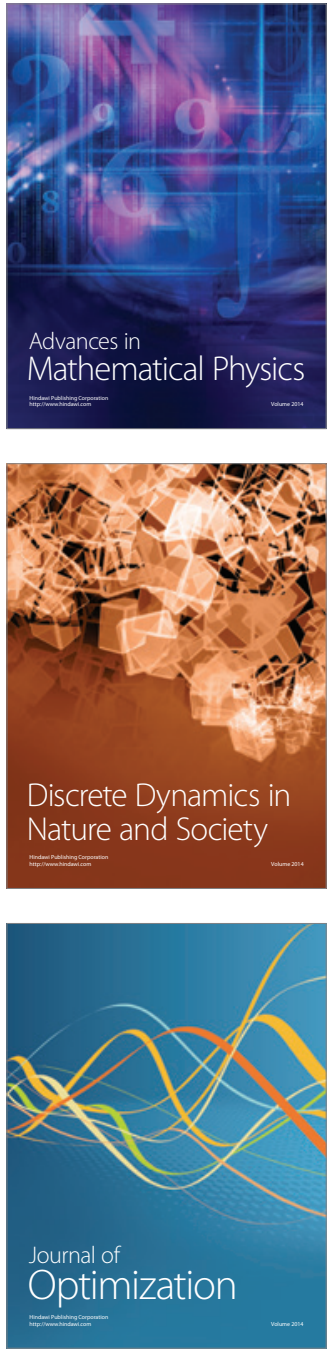